\title{
Tension development and muscle activation in the leg during gait in spastic hemiparesis: independence of muscle hypertonia and exaggerated stretch reflexes
}

\author{
W BERGER, G HORSTMANN, V DIETZ \\ From the Department of Clinical Neurology and Neurophysiology, University of Freiburg, Freiburg, West \\ Germany
}

SUMMARY In 15 patients with spastic hemiparesis the development of tension of calf muscles in relation to their electrical activation and their stretching period was studied on both sides during locomotion. Only in the spastic leg did isolated small biphasic potentials appear in the gastrocnemius E.M.G. with monosynaptic latency at the beginning of the stance phase, while the remaining gastrocnemius activation was reduced compared to the unaffected side. Perturbations of gait were followed in the spastic leg by a large monosynaptic response, while the polysynaptic reflex response was reduced. In the unaffected leg only a strong polysynaptic response appeared, which suggests a reciprocal modulation of monosynaptic and polysynaptic reflex responses. Tension development paralleled the gastrocnemius E.M.G. in the unaffected leg, while in the spastic leg tension was more closely correlated to muscle stretch. It is concluded that in spasticity the exaggerated monosynaptic reflexes represent only a small part of leg extensor activation during gait and that the tension development does not depend on these reflexes.

Exaggerated activity of $\alpha$ ( $\alpha$-spasticity) and $\gamma$-motoneurons ( $\gamma$-spasticity) is the most accepted hypothesis for muscle hypertonia in spasticity. ${ }^{1}$ However, a study of the active complex movements which are most impaired by muscle hypertonia, could not be found either in the older literature ${ }^{2}$ or in the more recent reports. ${ }^{3-5}$ It has been proposed that changes of mechanical muscle fibre properties contribute to muscle hypertonia. ${ }^{45}$

While earlier papers were concerned with the activity pattern of the spastic leg muscles during gait, no information about quick compensatory movements following disturbances of gait, and only indirect evidence for the generation of the abnormal tension development, was available.

The aim of this study was to analyse the activity and functional significance of mono- and polysynaptic reflexes during normal and disturbed gait, and to

Address for reprint requests: Dr W Berger, Abtlg. klinische Neurologie und Neurophysiologie, Hansastr. 9, D-7800 Freiburg, West Germany.

Received 20 September 1983 and in revised form 29 February 1984.

Accepted 10 March 1984 evaluate their contribution to the development of tension in the triceps surae to compensate for body load after impact. Hemiparetic patients were selected for this study in order to compare the unaffected leg with the spastic one of the same subject.

\section{Methods}

Fifteen patients with spastic hemiparesis were studied during slow gait $(1.5$ to $2.5 \mathrm{~km} / \mathrm{h})$ on a treadmill. Eleven of the patients suffered from a unilateral ischaemic infarction, while in the remainder had different causes (encephalitis 2, tumour 1 , and trauma 1 ). The age of the patients ranged from 39 to 74 years (mean 54 years). The spastic paresis had been present from between 4 months to 13 years (mean 2.9 years) and all patients exhibited exaggerated tendon reflexes and a positive Babinski sign on the affected side. The patients were able to stand and walk unaided or with minimal support.

The E.M.G. of the medial part of the gastrocnemius and of tibialis anterior of both legs was recorded using surface electrodes. Ground contacts of the heel and the ball of the foot as well as the beginning of the swing phase, were indicated separately by electrical switches fixed in the shoes. Joint angle changes at the ankle were recorded by a potentiometer. The changes in tension of the Achilles tendon during gait were measured by a buckle-type gauge. 
The gauge was fixed laterally near the tendon, while the tendon was pressed against the force-measuring branch by a metal frame from the contralateral side. The proportion of tension measured by the strain gauge amounted to about $3 \%$ of the total tension acting on the Achilles tendon and it increased in healthy subjects linearly with the gastrocnemius E.M.G.

Three patients were randomly displaced at distinct phases of the step cycle (in most cases at heel contact). For this, a step change of treadmill velocity (for example 1.5 $\mathrm{km} / \mathrm{h}$ to $7.5 \mathrm{~km} / \mathrm{h}$ in $100 \mathrm{~ms}$ ) was induced. For comparison, such an acceleration impulse was also applied in a standing position on the treadmill $(0$ to $6 \mathrm{~km} / \mathrm{h}$ in $100 \mathrm{~ms})$.

\section{Data processing}

The E.M.G. signals were recorded by a telemetric device (Schwarzer model 4024 FS) and filtered (time constant $3 \mathrm{~ms}$ ). It was ascertained that mechanical artifacts were minimal by passively shaking the legs and by monitoring the E.M.G. on an ink recorder during the experiment. The rectified E.M.G. signals, together with signals of a goniometer, electrical switches and the gauge on the Achilles tendon were averaged on line (Nicolet model 1072) using methods described in previous papers. ${ }^{46}$

For better comparison, the histogram of each patient was normalised to a one step cycle. The change of tension, developing during the stance phase in each patient, was the same on both sides, which was to be expected because the body weight is alternatively supported by each leg. It varied however from one subject to the other depending on the body weight and local factors at the Achilles tendon. Therefore the peak tension reached during stance was normalised to one unit for all patients. The electrical switches in the shoes were used as trigger pulses indicating the onset of displacement. The resolution of the averaged histogram amounted to 256 points for each step cycle. All averaged recordings were transferred to a Sirius Computer System and stored on a disk for normalisation of the individual histograms to a one step cycle, further processing and statistical analysis (for example mean values and standard deviations).

\section{Results}

\section{Gait pattern}

In fig 1 the gait histograms of 15 patients with spastic hemiparesis are summarised to show the factors determining the tension development in both legs. On the unaffected side, the peak increase in gastrocnemius E.M.G. occurred in the middle of the stance phase. The tension development paralleled the increase in E.M.G. amplitude and reached its maximum about $100 \mathrm{~ms}$ after the E.M.G. peak. With the plantarflexion of the foot at the end of the stance phase, the E.M.G. as well as the tension dropped to zero. During the swing phase, the foot was actively dorsiflexed by the tibialis anterior, but this was associated with only a small increase in tension. On the spastic side, the mean amplitude of ankle joint movement was about half that of the unaffected side. However, the stretch of the triceps surae during the passive dorsiflexion of the foot was not connected with an additional increase of gastrocnemius E.M.G. Except for a large synchronised E.M.G. peak, which appeared just after ground contact by the ball of the foot, that is at the onset of muscle stretch, the gastrocnemius activation remained on about the same level during the whole stretching period. The maximal gastrocnemius activation was reduced to about half of normal strength. In the individual histograms, the reduction of E.M.G. strength was correlated with the severity of paresis in each patient. In a few patients, as well as the gastrocnemius, the soleus and tibialis posterior-E.M.G.s were recorded. They showed, however, no essential difference from the gastrocnemius activation. The tension development closely paralleled the ankle joint movement through the whole stance phase, while the synchronised E.M.G. peak was not reflected in the tension trace. During the swing phase, the foot was only a little dorsiflexed despite the tibialis anterior activation and the gastrocnemius was already activated before ground contact (as seen in healthy subjects during tip toeing).

\section{Perturbation of gait}

Figure 2 shows the different compensatory reactions of both legs to a treadmill acceleration during gait and when standing still. To demonstrate the consistency of the responses, several single raw E.M.G traces were displayed with a higher resolution. The treadmill acceleration caused a quick dorsiflexion of the foot which resulted in a stretching velocity of $250-300 \%$ s (calculated from the goniometer trace). When the acceleration was induced during gait at heel contact of the unaffected leg (fig 2a) a strong gastrocnemius response appeared with a latency of $65-75 \mathrm{~ms}$ in respect to the onset of ankle joint movement (duration about $100 \mathrm{~ms}$ ); when the same stimulus was applied to the spastic leg only small biphasic potentials appeared with a latency of about $40 \mathrm{~ms}$ while the activity following these potentials was reduced compared to the unaffected leg. When the same impulse was induced while standing still (treadmill velocity $=0$; fig $2 \mathrm{~b}$ ) isolated biphasic potentials appeared in the gastrocnemius E.M.G. of both legs and with the same latency of $40 \mathrm{~ms}$, however, larger on the spastic side. On the unaffected side, these small potentials were followed by a strong gastrocnemius activation (latency about 80 $90 \mathrm{~ms}$ ), separated by an E.M.G. depression lasting about $35-40 \mathrm{~ms}$. In contrast to this, on the spastic side only a little background activity could be distinguished in this time period. Despite the small gas- 


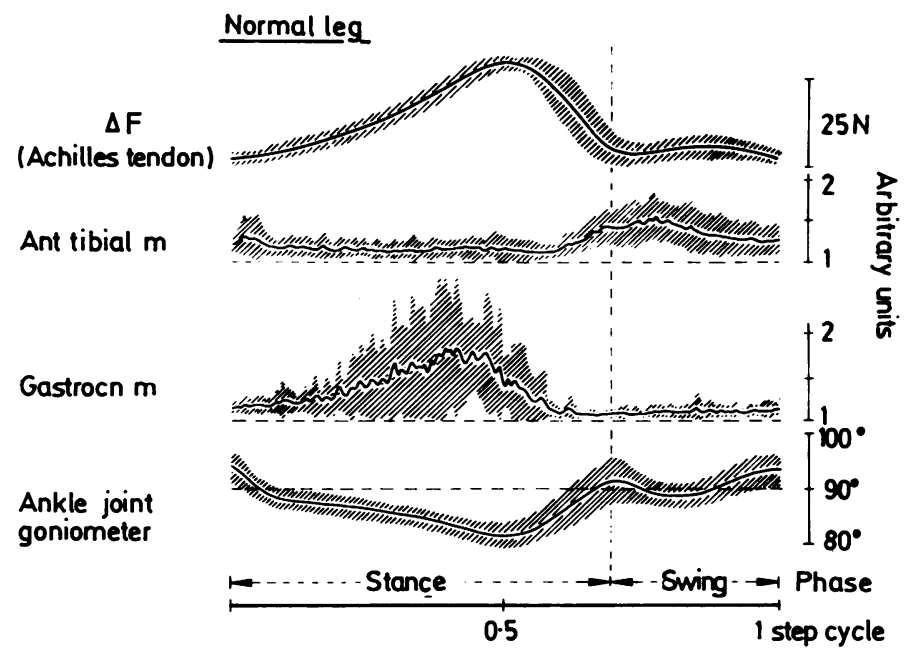

Spastic leg

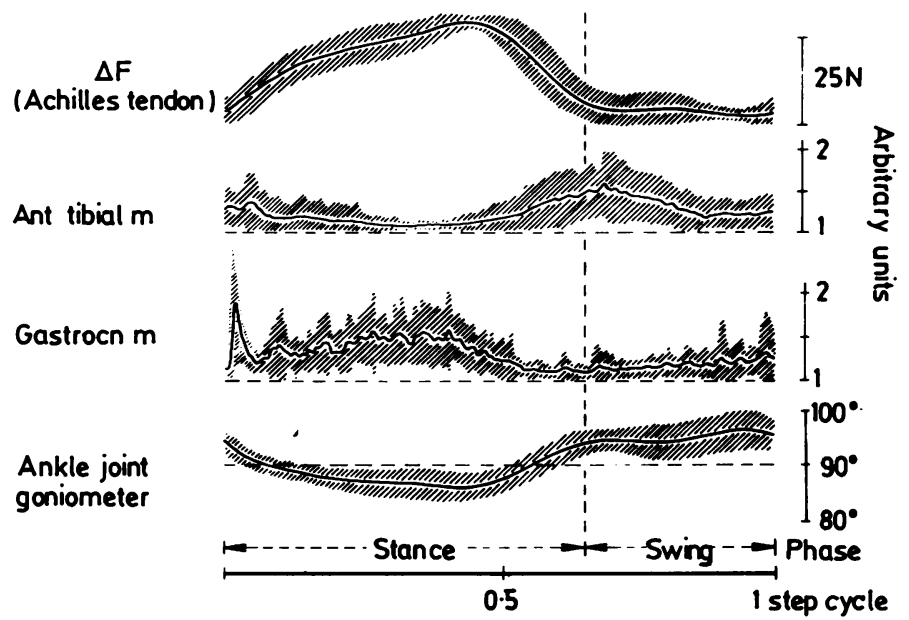

Fig 1 Gait pattern of the unaffected (upper trace) and the spastic leg (lower trace) in patients with spastic hemiparesis. Mean values and standard deviations of the averaged ( $n=32$ step cycles) histograms of 15 patients. The individual histograms were normalised to 1 step cycle. In each figure from top: Changes in tension at the Achilles tendon, rectified tibialis anterior and gastrocnemius EMG and ankle joint movements. 1 arbitrary unit equals about $1 \mathrm{mV}$.

trocnemius activation in the spastic leg the increase in tension at the Achilles tendon was the same on both sides. However, on the unaffected side the peak-tension was connected with the maximal E.M.G. amplitude while on the spastic leg it was connected with the maximal passive dorsiflexion of the foot, that is stretch of triceps surae.

\section{Discussion}

On the basis of the results two aspects will be discussed: the functional significance of mono- and polysynaptic reflex responses and, as a consequence of this, their contribution to the tension development in spastic gait. 

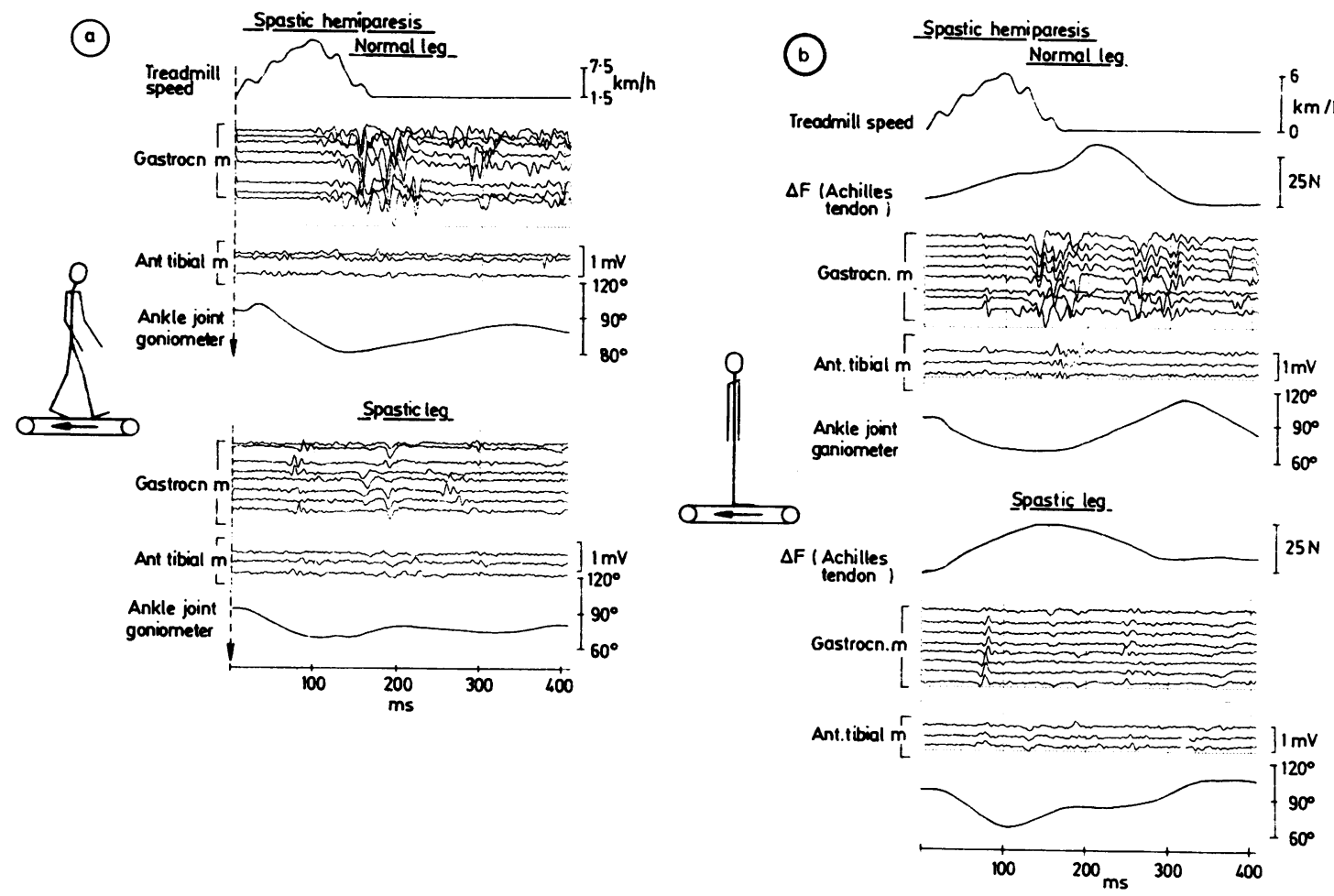

Fig 2 Leg muscle E.M.G. responses on the unaffected (upper trace) and spastic (lower trace) leg following a short treadmill acceleration during gait $(2 a)$ and standing $(2 b)$ of a patient with spastic hemiparesis (42 years old, unilateral cerebral haemorrhage 4 years previously). During gait the perturbation was induced randomly at heel contact of, respectively, the unaffected and spastic leg. Single gastrocnemius (8) and tibialis anterior (3) raw E.M.G. responses are displayed with a high gain, together with the treadmill speed and the averaged $(n=8)$ ankle joint movements. Arrow $(\downarrow)$ indicates ground contact. On the right side the averaged $(n=8)$ changes of tension at the Achilles tendon are shown.

\section{Reflex behaviour}

The early biphasic gastrocnemius E.M.G. potentials, which, on the basis of their latency, obviously represent monosynaptic stretch reflexes, frequently appear at the beginning of normal or disturbed stance phase on the spastic side. They do not arise on the unaffected side, although (especially during the acceleration impulse), stretch velocity of triceps surae should be high enough to evoke monosynaptic responses. Gottlieb and Agarwal ${ }^{7}$ demonstrated this in their experiments with a torque motor. This lack of monosynaptic responses suggests that, in healthy subjects, the monosynaptic response is inhibited during gait. Consequently monosynaptic responses did appear on the unaffected leg when the perturbations were applied during standing. This agrees with a recent study which showed that the $\mathrm{H}$ reflex was facilitated more during voluntary contractions than during walking. ${ }^{8}$
Load compensation following a perturbation was provided on the unaffected side by an "early" strong gastrocnemius activation (most probably corresponding to a polysynaptic reflex response). On the spastic side this leg extensor activation following the isolated biphasic potentials was always reduced, which corresponds to a reduction of the early polysynaptic response reported for torque motor perturbations applied to a spastic arm. ${ }^{9}$ This indicates that there exists a reciprocal modulation of monosynaptic and polysynaptic E.M.G. responses during gait, with the appearance of (normally suppressed) monosynaptic potentials and a reduction of the normally functionally essential polysynaptic reflex response in subjects with impaired function of supraspinal motor centres (both spastic patients and children around 1-2 years of age (unpublished observations)). The latency of the polysynaptic reflex response described here. which is about the 


\section{Muscle hypertonia and stretch reflexes}

same as that of the, so called, "M2-response" obtained in wrist displacements (around $60 \mathrm{~ms}$ ), makes it more likely that the former is mediated by a polysynaptic spinal than by a transcortical pathway, as has been suggested for the latter. This fact also supports the notion, that there exist different mechanisms for the long latency reflex responses obtained in different muscles. ${ }^{10 ~} 1$

\section{Tension development}

There is no obvious connection between the exaggerated monosynaptic reflexes and muscle hypertonia in spasticity, as has been assumed since Romberg. ${ }^{12}$ The tension development of the leg extensor muscles to support the body weight occurs with the stretching of the slightly tonically activated triceps surae, while it normally depends on the electrical muscle activation. The exaggerated monosynaptic reflexes obviously do not contribute much to muscle tension. Therefore, muscle hypertonia cannot be explained by enhanced motoneuron activity, but is probably due, as was recently suggested, ${ }^{45}$ to behavioural changes of lower motor unit activity resulting from the impaired supraspinal input, with consequent changes in mechanical muscle fibre properties.

We are grateful to Mr Stuart Fellows for correcting the English text.

This work was supported by the Deutsche Forschungsgemeinschaft-Sonderforschungsbereich 70 (Hirnforschung und Sinnesphysiologie) and Project "Entwicklungsmotorik" (Be 936/1-1)

\section{References}

'Herman R. The myotatic reflex. Clinicophysiological aspects of spasticity and contracture. Brain 1970;93:273-312.

${ }^{2}$ Altenburger H. Elektrodiagnostik. In: Bumke O, Foerster $\mathrm{O}$, eds. Handbuch der Neurologie. Hrsg. Berlin: Julius Springer. Allgemeine Neurologie 1937;III:968-1019.

${ }^{3}$ Knutsson E, Richards C. Different types of disturbed motor control in gait of hemiparetic patients. Brain 1979; 102:405-30.

${ }^{4}$ Dietz V, Quintern J, Berger W. Electrophysiological studies of gait in spasticity and rigidity. Evidence that altered mechanical properties contribute to hypertonia. Brain 1981;104:431-49.

${ }^{5}$ Dietz V, Berger W. Normal and impaired regulation of muscle stiffness in gait: a new hypothesis about muscle hypertonia. Ex Neurology 1983;79:680-7.

${ }^{6}$ Dietz V, Schmidtbleicher D, Noth J. Neuronal mechanisms of human locomotion. $J$ Neurophysiol 1979; 42: 1212-22.

7 Gottlieb GL, Agarwal GC. Response to sudden torques about ankle in man: myotatic reflex. $J$ Neurophysiol 1979;42:91-106.

" Morin C, Katz R, Mazieres L, Pierrot-Deseilligny E. Comparison of soleus $\mathrm{H}$ reflex facilitation at the onset of soleus contractions produced voluntarily and during the stance phase of human gait. Neurosci Lett 1982;33:47-53.

${ }^{9}$ Lee RG, Tatton WG. Long loop reflexes in man: clinical applications. Cerebral Motor Control in Man: Long Loop Mechanisms. In: Desmedt JE, ed. Basel: Karger. Progress in Clin Neurophysioly 1978;4:320 33.

${ }^{10}$ Berardelli A, Hallett M, Kaufmann C, Fome E, Berenberg W, Simon SR. Stretch reflexes of triceps surae in normal man. J Neurol Neurosurg Psychiatry 1982;45:513-25.

" Berardelli A, Sabra AF, Hallett M, Berenberg W, Simon S. Stretch reflexes of triceps surae in patients with upper motor neuron syndromes. J Neurol Neurosurg Psychiatry 1983;46:54-60.

${ }^{12}$ Romberg MH. Lehrbuch der Nervenkrankheiten des Menschen, 1851. Berlin: Verlag Alexander Duncker. 